\title{
Software Engineering Approaches to Semantic Web
}

\author{
J. S. Dong \\ National University of Singapore
}

\section{Overview}

In recent years, researchers have begun to explore the potential of associating web content with explicit meaning so that the web content becomes more machine-readable and intelligent agents can retrieve and process information readily. The Semantic Web (SW) [1] proposed by W3C is one of the most promising and accepted approaches. It may have great impacts to the current web. SW not only emerges from the Knowledge Representation and the Web Communities, but also brings the two communities closer together. The Software Engineering community can also play an important role in the Semantic Web development. Modeling and verification techniques can be useful at many stages during the design, maintenance and deployment of Semantic Web ontology. We believe Semantic Web will be a new research and application domain for software modeling techniques and tools.

In the development of Semantic Web there is a pivotal role for ontology, since it provides a representation of a shared conceptualisation of a particular domain that can be communicated between software programs. As autonomous software web agents may need to make their own decisions based on their knowledge, it is essential that the shared ontology is consistent. Ontology reasoners such as RACER and $\mathrm{FaCT}$ have been developed to reason ontologies with a high degree of automation. However, complex ontologyrelated properties may not be expressible within the current web ontology languages, consequently they may not be checkable by RACER and FaCT. We propose to use the software engineering techniques and tools, i.e., Z/EVES [9] and Alloy Analyzer(AA) [8], to complement the ontology tools for checking Semantic Web documents $[4,5]$. In our approach, Z/EVES is first applied to remove trivial syntax and type errors of the ontologies. Next, RACER is used to identify any ontological inconsistencies, whose origins can be traced by Alloy Analyzer. Finally Z/EVES is used again to express complex ontology-related properties and reveal errors beyond the modeling capabilities of the current web ontology languages. We have successfully applied this approach to checking a set of military plan ontologies.

This tutorial will include:

\author{
D. Dan \\ Power Automation Pte Ltd
}

- An introduction to Semantic Web languages (RDF and OWL [2]) and Semantic Web tools (FaCT[7] and RACER[6]).

- An introduction to software modeling techniques $\mathrm{Z}$ and Alloy and a demonstration on how they can facilitate modeling Web ontologies.

- A combined approach [3] to check and reasoning Web ontologies and related properties using the combination of RACER, Z/EVES [9] and the Alloy Analyser [8] tools.

\section{References}

[1] T. Berners-Lee, J. Hendler, and O. Lassila. The semantic web. Scientific American, May 2001.

[2] M. Dean and G. Schreiber (editors). OWL Web Ontology Language Reference. W3C Recommendation, http://www.w3.org/TR/owl-ref/, 2004.

[3] J. S. Dong, C. H. Lee, H. B. Lee, Y. F. Li, and H. Wang. A Combined Approach to Checking Web Ontology. In The 13th International World Wide Web Conference (WWW'04), refereed papers track. ACM Press, May 2004.

[4] J. S. Dong, C. H .Lee, Y. F. Li, and H. Wang. Verifying DAML+OIL and Beyond in Z/EVES. In The 26th International Conference on Software Engineering (ICSE'04). ACM/IEEE Press, May 2004.

[5] J. S. Dong, J. Sun, and H. Wang. Checking and Reasoning about Semantic Web through Alloy. In Proceedings of 12th Internation Symposium on Formal Methods Europe: FM'03, pages 796-813, Pisa, Italy, September 2003. LNCS, SpringerVerlag.

[6] Volker Haarslev and Ralf Möller. RACER User's Guide and Reference Manual: Version 1.7.6, December 2002.

[7] I. Horrocks. The FaCT system. Tableaux'98, Lecture Notes in Computer Science, 1397:307-312, 1998.

[8] D. Jackson, I. Schechter, and I. Shlyakhter. Alcoa: the alloy constraint analyzer. In Proc. 22nd International Conference on Software Engineering: ICSE'2000, pages 730-733, Limerick, Ireland, June 2000. ACM Press.

[9] M. Saaltink. The Z/EVES system. In J. P. Bowen, M. G. Hinchey, and D. Till, editors, ZUM'97: Z Formal Specification Notation, volume 1212 of Lecture Notes in Computer Science, pages 72-85. Springer-Verlag, 1997. 